
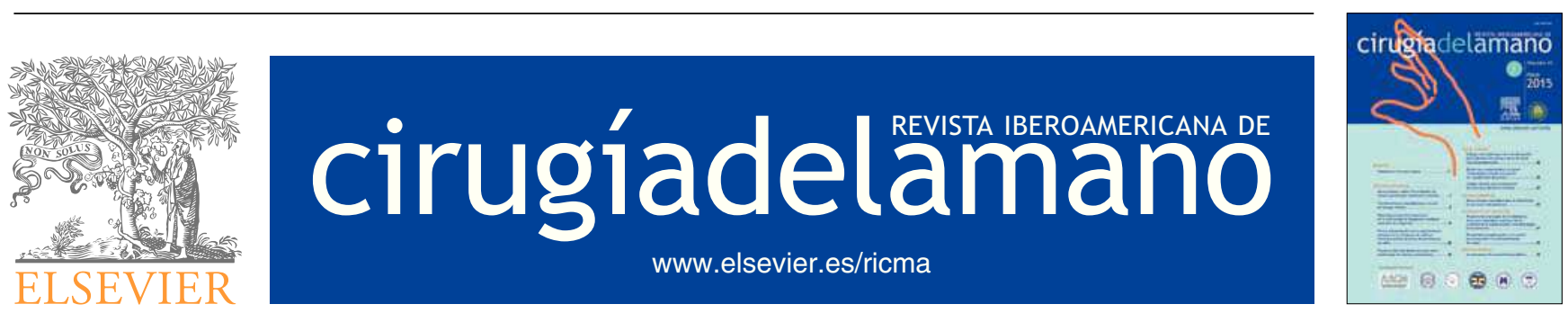

ARTÍCULO ORIGINAL

\title{
Fracturas del radio distal: encuesta sobre preferencias de manejo y tratamiento
}

\section{P.J. Delgado ${ }^{\mathrm{a}, *}$, D.M. Martínez-Capoccini ${ }^{\mathrm{b}}$ y J. Cerverac}

\author{
a Unidad de Cirugía de la Mano y del Miembro Superior, Hospital Universitario Madrid Montepríncipe, Universidad CEU \\ San Pablo, Boadilla del Monte, Madrid, España \\ b Servicio de Cirugía Ortopédica y Traumatología, Hospital Povisa, Vigo, España \\ c Servicio de Cirugía Ortopédica y Traumatología, Hospital General de Villalba, Collado Villalba, Madrid, España
}

Recibido el 1 de marzo de 2015. Aceptado el 1 de abril de 2015.

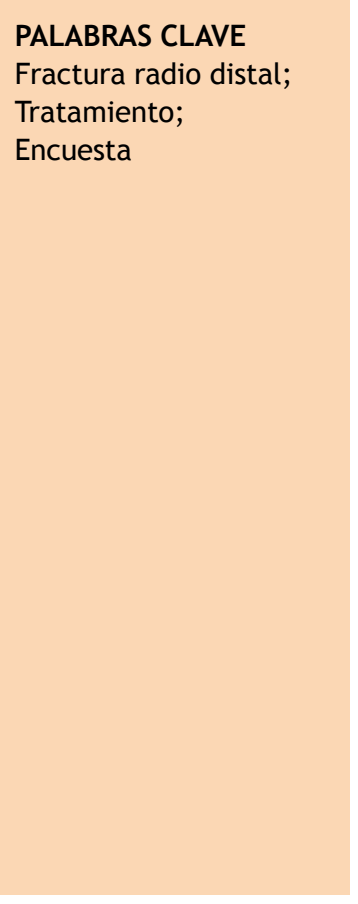

\begin{abstract}
Resumen
Objetivo: La fractura de la extremidad distal del radio (FEDR) es un traumatismo frecuente a cualquier edad y su tratamiento es aún tema de controversia. El objetivo del trabajo es determinar la tendencia actual de manejo en cirujanos especialistas en cirugía de la mano.

Material y método: Se realiza una encuesta electrónica remitida por correo electrónico a miembros de la Sociedad Española de Cirugía de la Mano (SECMA). A los participantes se les pregunta por sus preferencias respecto a clasificación, tratamiento conservador y quirúrgico de estas fracturas. Se valoran sus resultados con la evidencia científica actual al respecto.

Resultados: Se obtuvieron 49 respuestas (14,41\% de los socios encuestados) La mayoría tenían más de 21 años de experiencia como cirujanos de mano. La clasificación más usada fue la de AO-ASIF. Las FEDR estables se realiza de forma conservadora con yeso sin incluir el codo. El tratamiento de elección quirúrgico es la fijación interna con placas volares y tornillos. No es habitual la utilización de la artroscopia como asistente de la reducción y en casos de inestabilidad de la RCD se trata mediante fijación con agujas transfixiantes o tornillos de fijación de la estiloides cubital. No hay consenso de utilización de suplementos biológicos ni tratamiento coadyuvantes de la osteporosis. La toma de decisiones se realiza tomando en cuenta su experiencia personal y los conocimientos adquiridos durante su vida profesional.

Conclusiones: El manejo de las FEDR varía en función de la experiencia de cada cirujano y no se correlaciona necesariamente con la evidencia científica publicada al respecto. Su manejo es heterogéneo y se necesitan estudios mayores y elaborar patrones de consenso para el manejo de estas lesiones.
\end{abstract}

(c) 2015, SECMA. Publicado por Elsevier España, S.L.U. Este es un artículo Open Acces distribuido bajo los términos de la licencia CC BY-NC-ND (http://creativecommons.org/licenses/by-nc-nd/4.0/).

*Autor para correspondencia.

Correo electrónico: pedrojdelgado@me.com (P.J. Delgado).

(c) 2015, SECMA. Publicado por Elsevier España, S.L.U. Este es un artículo Open Acces distribuido bajo los términos de la licencia CC BY-NC-ND (http://creativecommons.org/licenses/by-nc-nd/4.0/). 


\section{KEYWORDS}

Distal radius fracture; Treatment; Survey

\section{Introducción}

Las fracturas del extremo distal del radio (FEDR) tienen en la actualidad una gran transcendencia social y personal, no solo en individuos en pleno desarrollo de sus actividades laborales, sino que también adquieren una importancia en la calidad de vida de personas de edad avanzada.

En los últimos años, las FEDR han aumentado su incidencia debido al aumento de la esperanza de vida, calidad de vida y las actividades de las personas. En EE. UU. se calcula que existen 37 millones de personas mayores de 65 años y España tiene una esperanza de vida de 81 años con una población que la mayoría sobrepasa los 65 años. Constituyen el $14 \%$ de todas las lesiones de la extremidad y el $17 \%$ de todas las fracturas tratadas en urgencias. Considerada la fractura más común de la extremidad superior en los individuos mayores de 65 años, representa hasta el 18\% de las fracturas que padecen, con una incidencia del $57-100 / 10.000$ pacientes al año ${ }^{1}$. La osteoporosis es el principal factor predisponente, y la caída el factor precipitante ${ }^{2}$. Las fracturas por osteoporosis siguen aumentando, produciendo un grave problema social, asistencial y económico ${ }^{3,4}$.

Debido al aumento de la calidad de vida y la expectativa de vida, en la actualidad nos encontramos ante una mayor demanda de buenos resultados con el tratamiento empleado para que les proporcionen una adecuada función de la muñeca, sin cambios significativos en los estilos de vida.

El objetivo principal de tratamiento, es la restitución anatómica lo más precisa posible para evitar la aparición de una artrosis postraumática. Las fracturas con una consolidación insuficiente o en mala posición tienen un efecto perjudicial sobre el funcionamiento de toda la articulación de la muñeca. Pero tampoco se ha encontrado una certeza de esto, ya que muchas fracturas con un mal resultado en cuanto a la reducción anatómica, suelen no presentar sintomatología ni limitaciones a la vida diaria ${ }^{5}$.

En cuanto al tratamiento quirúrgico, existe una relación de parámetros radiológicos que se consideran como definitorios de inestabilidad, que, a su vez, se asocian con mayor tendencia al desplazamiento secundario y menor probabilidad de conseguir resultados anatómicos con el tratamiento conservador. Estos criterios resultan válidos sobre todo para pacientes activos, en los que hay que ser muy riguroso en cuanto a los criterios de reducción, debiendo recurrir en muchas ocasiones al tratamiento quirúrgico para poder obtenerlos.

Hay descritas numerosas clasificaciones de estas fracturas y un amplio arsenal terapéutico para su tratamiento, que ha ido evolucionando a lo largo de la historia, aplicándose en función de escuelas o la propia experiencia de cada equipo, sin existir un aparente consenso de tratamiento y manejo de estas fracturas 6 .

El objetivo del trabajo es determinar las diferentes formas, tendencias y/o preferencias que existen en España en la actualidad, con respecto a la toma de decisiones en el tratamiento de las FEDR entre los especialistas de cirugía de la mano.

\section{Material y método}

Se realiza un estudio transversal en médicos especialistas en cirugía de la mano, todos socios de la Sociedad Española de Cirugía de la Mano (SECMA) a través de una encuesta on-line mediante la herramienta gratuita SurveyMonkey (www.sur- 
veymonkey.com). El estudio obtuvo el visto bueno de la SECMA, quien facilitó los correos de contacto de los socios, previa autorización y consulta de su junta directiva.

El cuestionario constaba de 10 preguntas sobre cómo realizaba cada profesional el manejo y tratamiento de las FEDR en su medio (tabla 1). La encuesta se realizó de forma anó- nima y voluntaria, quedando abierto el plazo de contestación desde el día 1 de febrero al 30 de junio del 2014. Se enviaron un total de 340 cuestionarios, de los cuales se obtuvieron 49 respuestas, lo que supone el $14,41 \%$ del total de encuestados. Los resultados obtenidos se contrastan con la evidencia científica publicada al respecto.

Tabla 1 Cuestionario de manejo de las fracturas del radio distal

1. Indique los años de experiencia como médico especialista en Cirugía Ortopédica y Traumatología:
a) Menos de un año
b) Entre 1-5 años
c) Entre 6-10 años
d) Entre 11-15 años
e) Entre 16-20 años
f) Más de 21 años

2. Indique, de entre las siguientes, la clasificación que utiliza con más frecuencia (puede marcar más de una opción):
a) AO-ASIF
b) Fernandez
c) Frykman
d) Melone
e) Epónimos (Colles, ...)
f) Ninguna

3. Ante una fractura del radio distal con criterios de inestabilidad, indique el tratamiento que más probablemente decidiría con los pacientes más habituales que usted trata:
a) Conservador
b) Quirúrgico

4. Cuando utiliza un yeso como tratamiento conservador, indique si inmoviliza el codo (pueden ser varias opciones):
a) Nunca
b) Siempre
c) Solo lo incluyo en fracturas inestables

5. En las fracturas del radio distal inestables, indique de entre las siguientes opciones, la que suele utilizar con más frecuencia dentro de su ámbito (admite más de una posibilidad):
a) Conservador
b) Reducción cerrada y fijación percutánea
c) Reducción cerrada y fijador externo
d) Reducción abierta y fijación interna con placa por vía volar
e) Reducción abierta y fijación interna con placa por vía dorsal

6. ¿Utiliza de forma habitual el artroscopio como asistente de la reducción?
a) No
b) Sí

7. En los casos que presentan una inestabilidad de la articulación radiocubital distal en la exploración tras haber realizado la fijación de la fractura del radio, ¿qué tratamiento adicional suele realizar? (puede utilizar varias opciones):
a) Ninguno
b) Sutura abierta del CFCT
c) Aguja de bloqueo radiocubital dista
d) Osteosíntesis de la estiloides cubital siempre que se pueda
e) Yeso braquipalmar

8. En los casos de pérdida ósea, ¿qué tratamiento adicional suele usar?:
a) Ninguno
b) Fosfato tricálcico
c) Hidroxiapatita 
Tabla 1 Cuestionario de manejo de las fracturas del radio distal (continuación)
d) Cemento óseo remodelable
e) Matriz ósea desmineralizada
f) Factores de crecimiento
g) Autoinjerto de cresta iliaca
h) Aloinjerto de banco de hueso

9. En pacientes con factores de riesgo de osteoporosis, indique si suele asociar un tratamiento antiosteoporótico:
a) No
b) Sí

10. Con respecto a cómo suele decidir el tratamiento de una fractura del radio distal, indique sobre qué suele basarse para realizar la elección (puede ser más de una opción):
a) Mi experiencia personal
b) Literatura publicada
c) Ambos

\section{Resultados}

Del total de encuestados, el 42,86\% tenían una experiencia media superior a los 21 años dedicados a la cirugía de la mano, seguido de los grupos que comprendían entre 11 a 15 años y 16 a 20 años, con 9 especialistas en cada grupo con un $18,37 \%$ ambos.

Los resultados se presentan en las figuras 1-10.

\section{Discusión}

Existen numerosas clasificaciones para identificar las fracturas de la extremidad distal del radio en la literatura. La clásica nominación en fracturas de Colles (extrarticular con desplazamiento dorsal) o de Smith (desplazamiento palmar), de Barton (desplazamiento de la fractura de la columna volar medial) o la fractura del "chauffeur" (desplazamiento de la estiloides radial), en la actualidad están en desuso. Lidström ${ }^{7}$ presentó una sistematización basada en el desplaza- miento, la afectación articular y la conminución, y Older ${ }^{8}$ clasificó las fracturas en 4 grupos basándose en el grado de desplazamiento, angulación dorsal, acortamiento del radio distal y presencia de conminución. En Norteamérica, Gartland y Wesley ${ }^{9}$ (1951) proponen tres simples grupos (Tipo 1: Fracturas extraarticulares; Tipo 2: Fracturas intraarticulares no desplazadas; Tipo 3: Fracturas articulares desplazadas); en los países escandinavos Frykmann ${ }^{10}$ (1967) diferencia las fracturas articulares y extraarticulares y examinó la fractura asociada de la estiloides cubital. En Francia, Castaing ${ }^{11}$ (1964) se basa en el mecanismo de la fractura, el desplazamiento y el número de fragmentos distales. Melone ${ }^{12}$ creó una clasificación orientada al tratamiento y fundamentada en el mecanismo de lesión y el grado de afectación del radio distal, excluyendo el cúbito distal y focalizando la gran importancia de la carilla articular medial. Cooney ${ }^{13}$ (1993) y Rayhack $^{14}$ (1990) valoran la afectación articular, la estabilidad y la reductibilidad de la fractura. La Clínica Mayo-Rochester, desarrolló una subclasificación de las fracturas intraarticulares en función de la reductibilidad de las mismas con ligamentotaxis. Posteriormente, el grupo AO elabo-

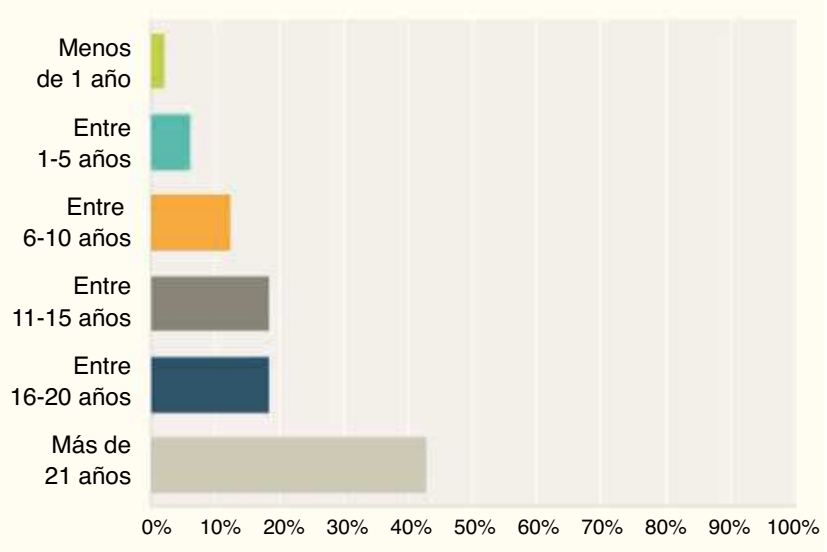

Figura 1 Indique los años de experiencia como médico especialista en Cirugía Ortopédica y Traumatología. 


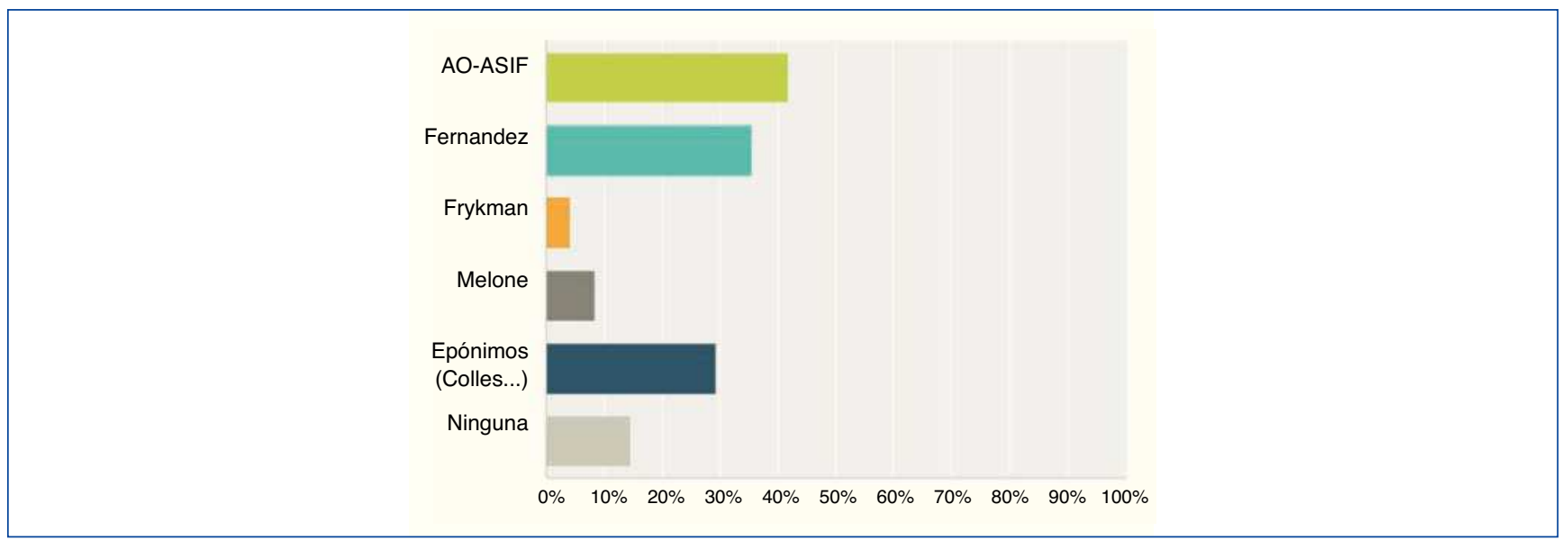

Figura 2 Clasificación que utiliza con más frecuencia.

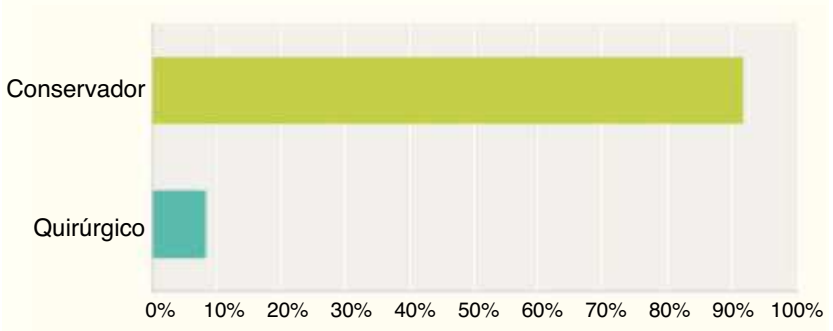

Figura 3 Ante una fractura de radio distal con estabilidad, indique el tratamiento que más probablemente decidiría con los pacientes más habituales que usted trata.

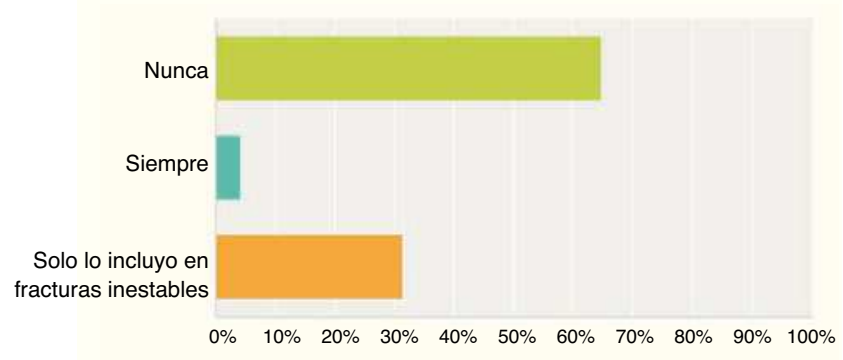

Figura 4 Cuando utiliza un yeso como tratamiento conservador, indique si inmoviliza el codo.

ró en 1986 una clasificación que es revisada en 1990 y 1995 , sobre la base de clasificaciones previas, a la vez que concibe la gravedad de la lesión de acuerdo con el grado de afectación intraarticular y de conminución metafisaria. Diferentes estudios han analizado la consistencia de las distintas clasificaciones, de las que se extrae y se percibe una concordancia interobservador moderada en la clasificación de Mayo y reducida en las Frykman, Melone y $\mathrm{AO}^{15}$. La clasificación de Fernández ${ }^{16}$ distribuye las fracturas en función del mecanismo de lesión, permitiendo valorar la presencia de lesiones ligamentosas, de los huesos del carpo, de la articulación radiocubital distal y de las partes blandas asociadas y seleccionar las opciones de tratamiento desde un punto de vista biomecánico. La clasificación de la AO es la que ha salido más utilizada por los cirujanos encuestados en este trabajo, con un $41,67 \%$ de seguimiento, siguiéndole en frecuencia de uso la Clasificacion de Fernández 35,42\%. Si bien hay muchas clasificaciones publicadas, ninguna es universal ni permite resolver todos los problemas. Las clasificaciones han variado durante el pasar de los años, se ha encontrado que 


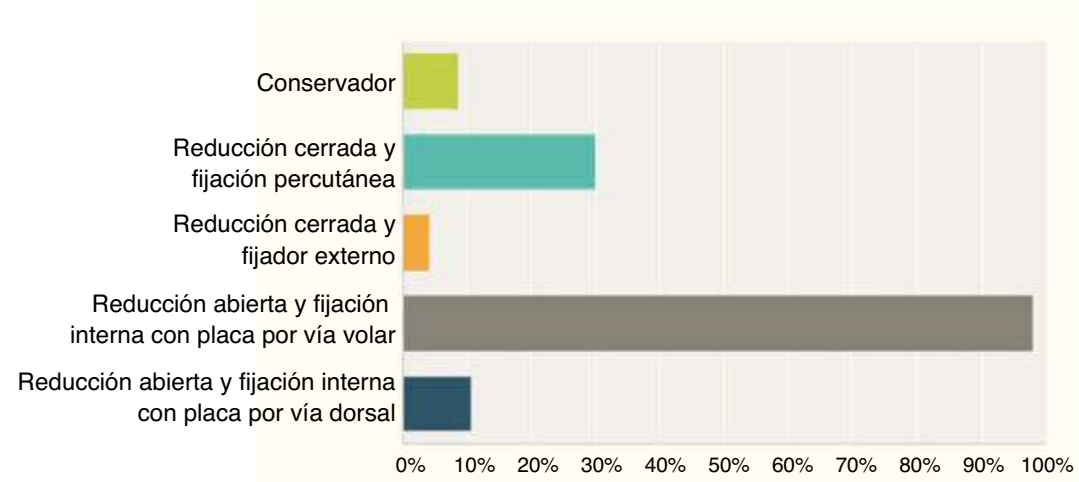

Figura 5 En las fracturas de radio distal inestables, indique de entre las siguientes opciones, la que suele utilizar con más frecuencia dentro de su ámbito.

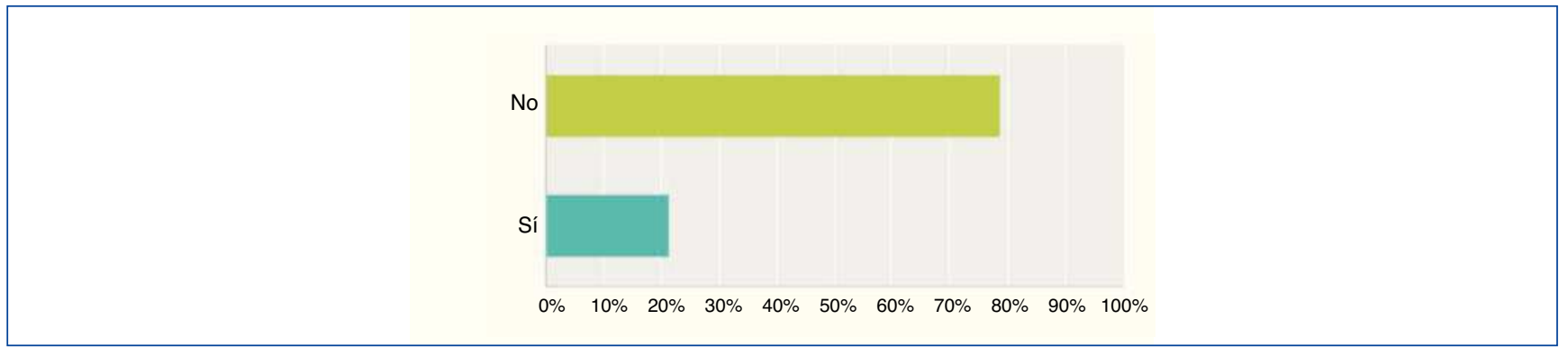

Figura 6 ¿Utiliza de forma habitual el artroscopio como asistente de la reducción?

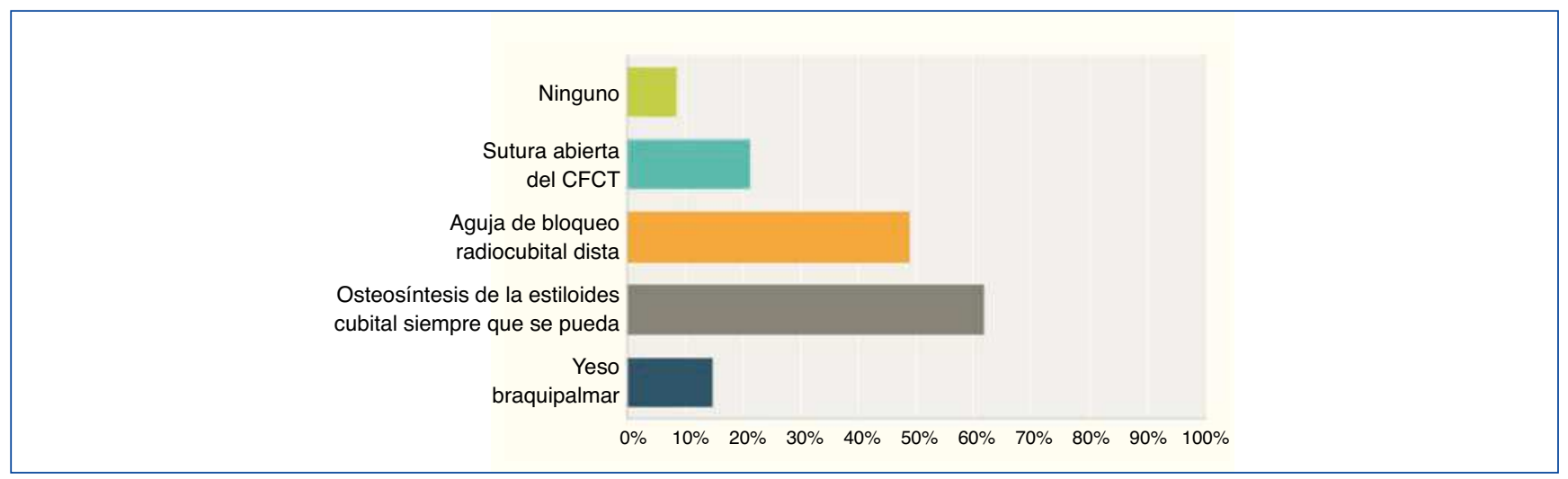

Figura 7 En los casos que presenta una inestabilidad de la articulación radiocubital distal en la exploración tras haber realizado la fijación de la fractura del radio, ¿qué tratamiento adicional suele usar?

todos son sistemas complejos y no estandarizados, que falta fiabilidad intraobservador e interobservador y falta de información pronóstico ${ }^{17,18}$.

El tratamiento inicial en urgencias de una FEDR con desplazamiento es la reducción cerrada del foco y estabilización con yeso, tras la cual deberían repetirse las radiografías para identificar la deformidad residual, aplicando las mediciones utilizadas para la valoración anatómica del extremo distal del radio (escalones articulares superiores a $2 \mathrm{~mm}$, acortamiento del radio distal $>3 \mathrm{~mm}$, inclinación dorsal $>10^{\circ}$ ), la cual tiene relación directa con el desarrollo de artrosis radiocarpiana y pobres resultados funcionales, siendo de interés al evaluar los resultados post-reducción y post-operatorios si fuese necesario ${ }^{19}$.

En el estudio realizado, ante una fractura del radio distal con criterios de estabilidad, el tratamiento de elección es el conservador en el $97,61 \%$ (44 respuestas) y solo procederían a una resolución quirúrgica en el $8,33 \%$ (solo 4 respuestas), probablemente asociado a centros relacionados con accidentes de trabajo o deportistas, en los que se pretende re- 


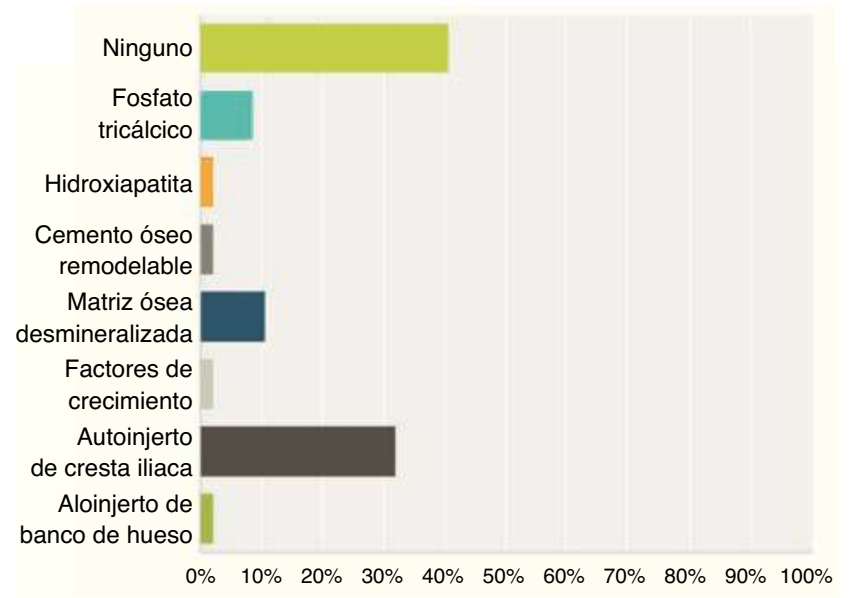

Figura 8 En los casos de pérdida ósea, ¿Qué tratamiento adicional suele utilizar?

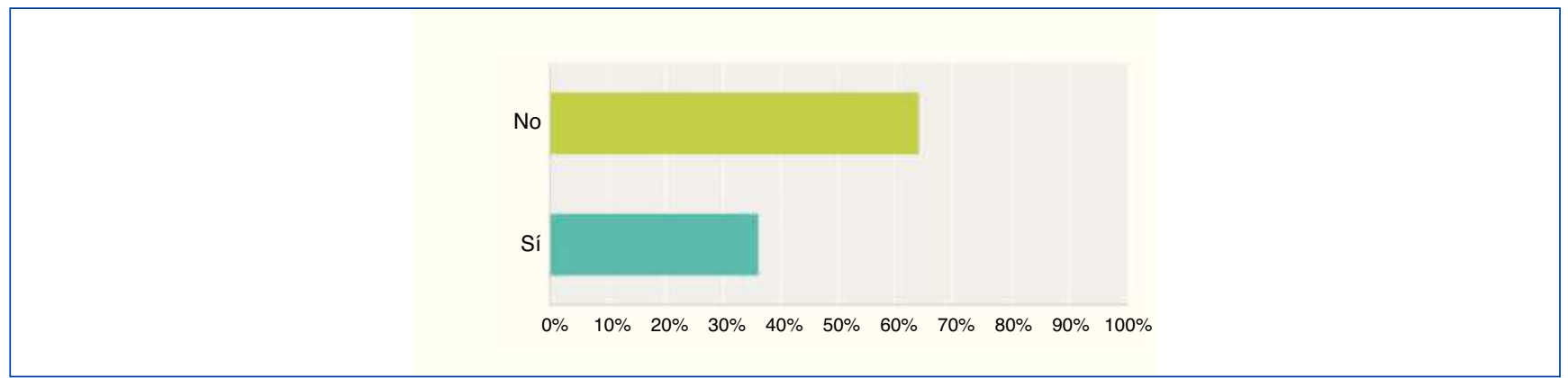

Figura 9 En pacientes con factores de riesgo de osteoporosis, indique si se suele asociar un tratamiento antiosteoporótico.

ducir el tiempo de recuperación. En consecuencia, el tratamiento de las FEDR se debe particularizar, de forma que, en muchos casos, pacientes con fracturas que por sus características son quirúrgicas, es más adecuado tratarlas de forma conservadora debido a las cualidades del paciente. Lo confirman los estudios de gran variedad de autores, consensuando el hecho de que, en pacientes de baja demanda y con escasa actividad manual, el tratamiento más apropiado sea el ortopédico. Por lo demás, este tratamiento no se basa únicamente en la reducción e inmovilización inicial, sino que requiere un estrecho seguimiento con el fin de detectar posibles desplazamientos secundarios y vigilar la correcta movilidad de las articulaciones vecinas. La pérdida de seguimiento de estos pacientes es frecuente. Tejwani y cols. ${ }^{20}$, en un estudio de 293 fracturas de extremidad distal del radio encontraron que la mayor prevalencia de pérdida de seguimiento (52-74\%) se daba en las fracturas extrarticulares, de baja energía y tratadas conservadoramente; es decir, en el perfil típico de la fractura del anciano.

Con respecto al tratamiento con yeso cerrado, en el $64,58 \%$ no incluía el codo en la inmovilización y el $31,25 \%$ solo lo hace cuando se encuentra ante fractura inestable. Si bien se recomendaba la utilización de un yeso por encima del codo en fracturas inestables, para mantener el antebra- zo en supinación y contrarrestar la acción del $m$. Brachiorradialis ${ }^{21,22}$, otros trabajos hacen referencia a una mayor actividad sobre la fractura del $m$. Pronator Quadratus y recomiendan su inmovilización en pronación ${ }^{23}$. En la actualidad está demostrado que estas posiciones realmente tengan influencia. Estudios prospectivos aleatorios no han encontrado diferencias a largo plazo entre la inclusión o no del codo en la inmovilización con yeso del antebrazo, ni siquiera con una inmovilización en forma de " $U$ " o en "lengua de gato"24,25. Tras la inmovilización con yeso, el paciente, sobre todo si es anciano, deberá, además, hacer ejercicios activos con los dedos de la mano, el codo y el hombro.

No hay duda de que el resultado funcional final es peor cuanta más deformidad residual exista. Se debe tener en cuenta que las fracturas de esta región anatómica tienen una elevada morbilidad. Si la consolidación no logra restituir la anatomía articular de la muñeca, lo habitual es que quede un déficit funcional con limitación de movilidad, deformidades, dolor residual y pérdida de fuerza. Según Frykmann ${ }^{10}$, entre un $2 \%$ y un $5 \%$ de malos resultados funcionales pueden existir pese a la obtención de resultados radiológicos anatómicos excelentes. Por otro lado, varios estudios informan de resultados funcionales excelentes con una consolidación anatómica no adecuada ${ }^{26}$ y alguno afirma que no 


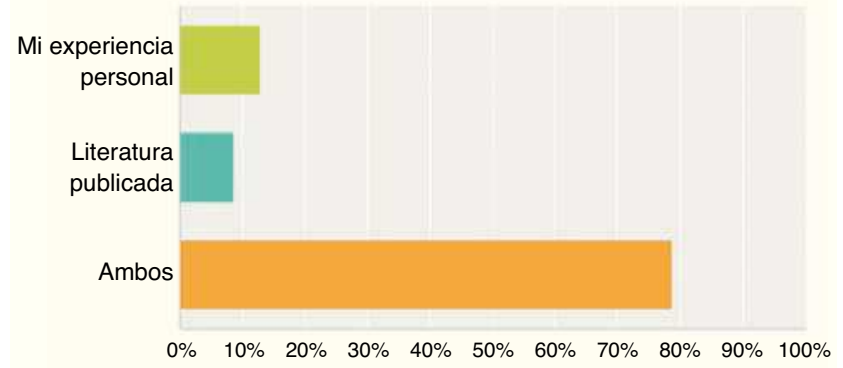

Figura 10 Con respecto a cómo suele decidir el tratamiento de una fractura de radio distal, indique sobre que suele basarse para la realización de la elección.

se correlaciona el resultado funcional con el resultado radiográfico y sí, en relación directa, con la actividad que el paciente solicite a su muñeca ${ }^{5}$.

Así pues, se debe individualizar el tipo de tratamiento, de forma que, al elegir el ortopédico, hay que tener en cuenta tanto las características de la fractura, en las que adquiere especial importancia la ausencia de criterios de inestabilidad, como las características particulares del paciente, atendiendo expresamente su situación funcional previa a la fractura, independiente de su edad cronológica, y a la presencia de comorbilidad asociada.

Existe una relación de parámetros radiológicos que se consideran como definitorios de inestabilidad, que, a su vez, se asocian con mayor tendencia al desplazamiento secundario y menor probabilidad de conseguir resultados anatómicos con el tratamiento conservador ${ }^{19}$. Sin embargo, no todas las indicaciones quirúrgicas vienen dadas por el tipo de fractura y su reducción, también hay que tener en cuenta al paciente, sus enfermedades, su edad y, sobre todo, su actividad manual y demanda funcional, que juegan un papel fundamental en la decisión del tratamiento definitivo. Mackenney y cols. ${ }^{27}$ han demostrado que los pacientes con más de 80 años de edad presentan más inestabilidad de la fractura y un mayor número de consolidación en mala posición, independientemente del grado de desplazamiento que presente.

El material de osteosíntesis, utilizado para el tratamiento quirúrgico de las fracturas, ha cambiado aceleradamente en los últimos años, modificándose todas las líneas de fijación interna en general. Existiendo así, diversas opciones de tratamiento quirúrgico, desde agujas percutáneas, a la fijación externa basada en el concepto de ligamentotaxis, y la osteosíntesis con placa, la cual es una mejora de métodos de fijación interna, fundamentalmente tras la aparición de placas bloqueadas de ángulo fijo y, posteriormente, de ángulo variable ${ }^{28}$. El gran objetivo de la osteosíntesis con reducción abierta y fijación interna con placa es conseguir una reducción anatómica de la superficie articular ${ }^{29,30}$. En nuestro trabajo, el método de fijación más utilizado para las fracturas inestables fue la reducción abierta y fijación con placa por vía volar $(97,87 \%)$. Múltiples trabajos han demostrado mejores resultados y grandes ventajas con el uso de las placas en el tratamiento de las FEDR en el anciano, fundamentalmente porque este tratamiento permite una correcta reducción de los fragmentos, proporciona una gran estabilidad incluso en huesos deficitarios, y, en muchos casos, retira la inmovilización postoperatoria más precozmente, acelerando la recuperación funcional y mejorando el tratamiento global de las mismas ${ }^{28,30,31}$. La posibilidad de bloquear los tornillos 0 pernos en los orificios de la placa la convierte en un bloque sólido que contribuye a puentear las fuerzas a nivel del foco de fractura. Las complicaciones asociadas a la fijación con placa volar son raras, y con frecuencia están relacionadas con la técnica quirúrgica ${ }^{32}$. Las lesiones de los tendones extensores son frecuentes, sobre todo el extensor largo del pulgar que ocurre en el $3 \%$ de los casos y se asocia a tornillos excesivamente largos, que protuyen a través de la cortical dorsal y penetran la vaina tendinosa, lesión que puede evitarse mediante una inspección cuidadosa bajo fluoroscopia en proyecciones laterales con inclinación cráneo-caudal a $20 \#$ y axial de la muñeca ("skyline-view") 33. Las lesiones de tendones flexores son menos frecuentes y, especialmente el del flexor largo del pulgar, se asocian al reborde distal epifisario de la placa, por lo que se recomienda cubrirlo con el músculo pronador cuadrado siempre que sea posible.

La artroscopia ha demostrado ser un complemento útil en el manejo de las FEDR. La reducción abierta y fijación con placa volar, método quirúrgico, es el más utilizado frente a FEDR inestables, aparentemente con mayor adhesividad por parte de los cirujanos y con posibles mejores resultados. Estudios prospectivos comparativos aleatorizados han demostrado resultados superiores (respecto a alineación radiológica, arcos de movilidad y resultados en las escalas funcionales) en pacientes tratados bajo artroscopia respecto a la fluoroscopia. Edwards y cols. ${ }^{34}$ compararon la reducción obtenida en 15 FEDR intraarticulares mediante artroscopia y fluoroscopia y/o proyecciones radiológicas convencionales. Si bien la magnitud del hundimiento fue medida de forma similar con ambos métodos, el 33\% de los casos en los que se interpretó con fluoroscopia y/o radiografías como una reducción óptima, al utilizar el artroscopio se objetivó una separación mayor a $1 \mathrm{~mm}$. En otro trabajo similar, Ruch y cols. ${ }^{35}$ en 30 casos de FEDR tratados con fijación externa, comprobaron una mejor valoración de las lesiones (especialmente en la vertiente ulnar) y un mejor rango de supinación y flexo-extensión en los casos tratados bajo artroscopia respecto a los que se utilizó solo la fluoroscopia. Otras ventajas de la utilización del artroscopio es la visualización directa de la "geografía” exacta de 
la fractura, la posibilidad de lavar el hematoma de la fractura que está en la articulación (precursor de la rigidez) así como identificar tornillos intraarticulares, en el caso de placas bloqueadas de ángulo fijo por vía volar, que pueden pasar inadvertidas en las proyecciones habituales de fluoroscopia en quirófano. Las FEDR pueden asociar lesiones del complejo fibrocartílago triangular (49-78\%), rotura del ligamento escafolunar (8-54\%) y lesiones del ligamento lunotriquetal $(12-16 \%)^{36}$. A pesar de sus ventajas, solo el $21,28 \%$ de los encuestados suele utilizar el artroscopio como asistente de la reducción. En la actualidad, en nuestro país, es de poco uso como ayuda el artroscopio ante la cirugía, lo cual puede estar en relación con su alta y larga curva de aprendizaje, o ante la incertidumbre de prolongación del tiempo quirúrgico.

La inestabilidad de la RCD es aún un tema controvertido de manejo en estas fracturas. Lindau y cols. publicaron en el año 2000 la necesidad de revisar las lesiones de FCT que se encontraban en pacientes con RCD inestable en el momento de la FEDR, ante el riesgo de inestabilidad residual ${ }^{37}$. Sin embargo, revisado este mismo grupo de pacientes 15 años después, en aquellos casos en los que no se realizó tratamiento de la lesión del FCT, solo un caso evidenció una inestabilidad sintomática de la RCD ${ }^{38}$. En los casos encuestados, esta circunstancia ha sido resuelta mediante sutura abierta de CFCT en el 21,28\%, agujas de bloqueo RCD en 48,94 , osteosíntesis de la estiloides cubital en el $61,70 \%$ y la colocación de un yeso braquiopalmar en el $14,89 \%$.

Otro tema en auge es el uso de injerto óseo en pacientes ancianos con osteoporosis tanto como los sustitutos óseos como los cementos han demostrado ser elementos que mejoran la fijación y, por ende, el éxito del tratamiento quirúrgico en las fracturas que presentan déficit óseo. Permanece por probarse cuál de los numerosos tipos de sustitutos de injertos pueden sustituir mejor el autoinjerto, mejorar los resultados, disminuir la necesidad de fijación y reducir el tiempo de consolidación en las fracturas distales de radio. Rajan y cols. ${ }^{39}$ publicaron el único estudio prospectivo comparativo aleatorio del uso de aloinjerto esponjoso e injerto de hueso autólogo, concluyendo que no existían diferencias entre ambos respecto a consolidación ósea y resultado funcional, salvo más rapidez de utilización y menor morbilidad de la zona donante en el caso de la utilización del aloinjerto. Al preguntar sobre el tratamiento adicional que se suele usar en los casos de pérdida ósea el resultado ha sido muy heterogéneo. El tratamiento más usado según las respuestas ha sido ninguno con un 40,43\% (19 respuestas), seguido de autoinjerto de cresta ilíaca 31,91\% (15 respuestas), luego el uso de matriz ósea desmineralizada con el 10,64\% (5 respuestas), el fosfato tricálcico con el $8,51 \%$ (4 respuestas) y con un porcentaje igual de $2,13 \%$ de uso (1 respuesta en cada uno obtenida) seguirían la hidroxiapatita, el cemento óseo remodelable, el uso de factores de crecimiento plaquetarios y el aloinjerto de banco de hueso. Ante un hueso de mala calidad osteoporótico, la mayoría no suele aportar sustituyentes que den más estabilidad al tratamiento, y que posteriormente un grupo moderado si suele ayudarse con autoinjerto de cresta iliaca.

En cuanto al uso de tratamiento anti-osteoporótico en pacientes con factores de riesgo de osteoporosis, el 63,83\% (30 respuestas) no suele indicarlo, y el $36,17 \%$ (17 respuestas) si lo utiliza. Aquellos con factores de riesgo osteoporóticos, el uso de los nuevos tratamientos está en minoría con respecto al no utilizar o administrar al paciente un ayudante formador de hueso.

Con respecto a en que suele basarse para la decisión del tratamiento de una fractura del radio distal, permitiéndose más de una opción, un $12,77 \%$ (6 respuestas) se basa en su experiencia personal, un $8,51 \%$ (4 respuestas) en la literatura publicada y el $78,72 \%$ (37 respuestas) suele basarse tanto en su experiencia como en la literatura. Esto puede indicar que en nuestro país, la mayoría de los cirujanos de la mano, trabajan tomando en cuenta su experiencia personal y los conocimientos adquiridos durante su vida profesional junto al hecho de mantenerse actualizados con las investigaciones, publicaciones y protocolos realizados por las diferentes sociedades americana o europea en cuanto al tema de FEDR, con poca adherencia hacia las publicaciones, y mayor adherencia al trabajar según la experiencia propia, lo cual dificulta llegar a un consenso. Esto también podría estar ligado al medio donde se trabaje, a los recursos que se puedan obtener para actualizarse en los tratamientos, y al tiempo que cada medio permite dedicar a cada caso.

El estudio presenta limitaciones, como el número de respuestas recibidas y el grado de subjetividad que comportan este tipo de estudios anónimos, por lo que no podemos extraer conclusiones definitivas. Si bien la participación en la encuesta ha sido baja (solo un 14\%), lo cual puede suponer la principal limitación del estudio, debemos tener en cuenta que aproximadamente el $70 \%$ de los casos pertenecían a profesionales de más de 10 años de experiencia en cirugía de la mano (el $41 \%$ por encima de 21 años), con lo que estos resultados pueden considerarse como una visión general de tendencias de tratamiento y manejo en nuestro país en el momento actual.

\section{Conclusiones}

En relación con las respuestas recibidas de los cirujanos participantes, podemos afirmar que en el grupo de estudio la clasificación más usada es la de la AO (probablemente por su mayor actualización y reproductibilidad) y que el tratamiento de las fracturas estables se realiza de forma conservadora con yeso que no suele subir por encima del codo. El tratamiento de elección quirúrgico es la fijación interna con placas volares y tornillos. No es habitual la utilización de la artroscopia como asistente de la reducción y en casos de inestabilidad de la RCD se trata mediante fijación con agujas transfixiantes o tornillos de fijación de la estiloides cubital. No hay consenso de utilización de suplementos biológicos ni tratamiento coadyuvantes de la osteporosis.

Por otra parte, la toma de decisiones se realiza tomando en cuenta la experiencia personal y los conocimientos adquiridos durante la vida profesional en gran parte de los casos. Este último aspecto puede estar en relación con los años de dedicación, detalle no objeto de este trabajo.

El manejo de las FEDR varía en función de la experiencia de cada cirujano y no se correlaciona necesariamente con la evidencia científica publicada al respecto. Su manejo es heterogéneo y se necesitan estudios mayores y elaborar patrones de consenso para el manejo de estas lesiones. 


\section{Conflicto de intereses}

Los autores declaran no tener ningún conflicto de intereses.

\section{Bibliografía}

1. MCQueen MM. Epidemiology of fractures of the radius and ulna. En: McQueen MM, Jupiter JB, editores. Radius and ulna. Musculoeskeletal trauma series. Oxford: Butterworth-Heinemann; 1999. p. 1-11.

2. Hegeman JH, Oskam J, van der Palen J, Ten Duis HJ, Vierhout PA. The distal radial fracture in elderly women and the bone mineral density of the lumbar spine and hip. J Hand Surg $[\mathrm{Br}]$. 2004;29:473-6.

3. Anzarut A, Johnson JA, Rowe BH, Lambert RG, Blitz S, Majumdar SR. Radiologic and patient-reported functional outcomes in an elderly cohort with conservatively treated distal radius fractures. J Hand Surg Am. 2004;29:1121-7.

4. Vogt MT, Cauley JA, Tomaino MM, Stone K, Williams JR, Herndon $\mathrm{JH}$; The Study of Osteoporotic Fractures. Distal radius fractures in older women: a 10-year follow-up study of descriptive characteristics and risk factors. The study of osteoporotic fractures. J Am Geriatr Soc. 2002;50:97-103.

5. Young BT, Rayan GM. Outcome following nonoperative treatment of displaced distal radius fractures in low-demand patients older than 60 years. J Hand Surg Am. 2000;25:19-28.

6. Diaz-Garcia RJ, Oda T, Shauver MJ, Chung KC. A systematic review of outcomes and complications of treating unstable distal radius fractures in the elderly. J Hand Surg Am. 2011;36:824-35.

7. Lidstrom A. Fractures of the distal end of the radius. A clinical and statistical study of end results. Acta Orthop Scand Suppl. 1959;41:1-118.

8. Older TM, Stabler EV, Cassebaum WH. Colles fracture evaluation and selection of therapy. J Trauma. 1965;5:469-76.

9. Gartland JJ Jr, Werley CW. Evaluation of healed Colles' fractures. J Bone Joint Surg Am. 1951;33-A:895-907.

10. Frykman G. Fracture of the distal radius including sequelae. Acta Orthop Scand. 1967; Suppl 108:3+.

11. Castaing J. (Le Club des Dix). Les fractures récentes de l'extrémité inférieure du radius chez l'adulte. Rev Chir Orthop Repar Appar Mot. 1964;50:581-666.

12. Melone CP Jr. Distal radius fractures: patterns of articular fragmentation. Orthop Clin North Am 1993;24:239-53.

13. Cooney WP. Fractures of the distal radius: a modern teratmentbased classification. Orthop Clin North Am. 1993;24:211-6.

14. Rayhack J. Symposium: management of intraarticular fractures of the distal radius. Contemp Orthop .1990;21:71-104.

15. Flikkilä T, Nikkola-Sihto A, Kaarela O, Pääkkö E, Raatikainen T. Poor interobserver reliability of $\mathrm{AO}$ classification of fractures of the distal radius. Additional computed tomography is of minor value. J Bone Joint Surg Br. 1998;80:670-2.

16. Fernández DL. Fractures of the distal radius: operative treatment. Instr Course Lect. 1993;42:73-88.

17. Naqvi SG, Reynolds T, Kitsis C. Interobserver reliability and intraobserver reproducibility of the Fernandez classification for distal radius fractures. J Hand Surg Eur Vol. 2009;34:483-5.

18. Andersen DJ, Blair WF, Steyers CM Jr, Adams BD, el-Khouri GY, Brandser EA. Classification of distal radius fractures: an analysis of interobserver reliability and intraobserver reproducibility. J Hand Surg Am. 1996;21:574-82.

19. Lafontaine M, Hardy D, Delince P. Stability assessment of distal radius fractures. Injury. 1989;20:208-10.

20. Tejwani NC, Takemoto RC, Nayak G, Pahk B, Egol KA. Who is lost to followup?: a study of patients with distal radius fractures. Clin Orthop Relat Res. 2010;468:599-604.
21. Sarmiento A, Pratt GW, Berry NC, Sinclair WF. Colles' fractures. Functional bracing in supination. J Bone Joint Surg Am. 1975;57:311-7.

22. Bünger C, Sølund K, Rasmussen P. Early results after Colles' fracture: functional bracing in supination vs dorsal plaster immobilization. Arch Orthop Trauma Surg. 1984;103:251-6.

23. Wahlström O. Treatment of Colles' fracture. A prospective comparison of three different positions of immobilization. Acta Orthop Scand. 1982;53:225-8.

24. Pool C. Colles's fracture. A prospective study of treatment. J Bone Joint Surg Br. 1973;55:540-4.

25. Bong MR, Egol KA, Leibman M, Koval KJ. A comparison of immediate postreduction splinting constructs for controlling initial displacement of fractures of the distal radius: a prospective randomized study of long-arm versus short-arm splinting. J Hand Surg Am. 2006;31:766-70.

26. Goldfarb CA, Rudzki JR, Catalano LW, Hughes M, Borrelli J Jr. Fifteen-year outcome of displaced intra-articular fractures of the distal radius. J Hand Surg Am. 2006;31:633-9.

27. Mackenney PJ, McQueen MM, Elton R. Prediction of instability in distal radial fractures. J Bone Joint Surg Am. 2006;88:1944-51.

28. Chung KC, Squitieri L, Kim HM. Comparative outcomes study using the volar locking plating system for distal radius fractures in both young adults and adults older than 60 years. J Hand Surg Am. 2008;33:809-19.

29. Synn AJ, Makhni EC, Makhni MC, Rozental TD, Day CS. Distal radius fractures in older patients: is anatomic reduction necessary? Clin Orthop Relat Res. 2009;467:1612-20.

30. Figl $M$, Weninger $P$, Jurkowitsch J, Hofbauer $M$, Schauer J, Leixnering $M$. Unstable distal radius fractures in the elderly patient-volar fixed-angle plate osteosynthesis prevents secondary loss of reduction. J Trauma. 2010;68:992-8.

31. Souza L, Maisonnave A, Kaempf R, Delgado PJ. Evaluación de la fisioterapia precoz en las fracturas de la extremidad distal del radio tratadas mediante placa volar de ángulo fijo. Trauma Fund Mapfre. 2008;20:29-37.

32. Trumble TE, Schmitt SR, Vedder NB. Factors affecting functional outcome of displaced intra-articular distal radius fractures. J Hand Surg Am. 1994;19:325-40.

33. Ozer K, Wolf JM, Watkins B, Hak DJ. Comparison of 4 fluoroscopic views for dorsal cortex screw penetration after volar plating of the distal radius. J Hand Surg Am. 2012;37:963-7.

34. Edwards CC 2nd, Haraszti CJ, McGillivary GR, Gutow AP. Intraarticular distal radius fractures: arthroscopic assessment of radiographically assisted reduction. J Hand Surg Am. 2001;26: 1036-41.

35. Ruch DS, Vallee J, Poehling GG, Smith BP, Kuzma GR. Arthroscopic reduction versus fluoroscopic reduction in the management of intra-articular distal radius fractures. Arthroscopy. 2004;20:225-30.

36. Delgado PJ, Figueredo FA, Gil de Rozas M, Truan JR. Tratamiento asistido por artroscopia de las fracturas del radio distal. Rev Esp Artrosc Cir Articul. 2014;1:1-8.

37. Lindau T, Adlercreutz C, Aspenberg P. Peripheral tears of the triangular fibrocartilage complex cause distal radioulnar joint instability after distal radial fractures. J Hand Surg Am. 2000;25:464-8.

38. Mrkonjic A, Geijer M, Lindau T, Tägil M. The natural course of traumatic triangular fibrocartilage complex tears in distal radial fractures: a 13-15 year follow-up of arthroscopically diagnosed but untreated injuries. J Hand Surg Am. 2012;37:155560.

39. Rajan GP, Fornaro J, Trentz O, Zellweger R. Cancellous allograft versus autologous bone grafting for repair of comminuted distal radius fractures: a prospective, randomized trial. J Trauma. 2006;60:1322-9. 